\title{
Current Issues in Multiple Domain Semantic Reconciliation for Ontology-Driven Interoperability in Product Design and Manufacture
}

\author{
Matheus Beltrame Canciglieri ${ }^{1}$, Anderson Luis Szejka ${ }^{1 \text { [0000-0001-8977-1351] }}$, Osiris \\ Canciglieri Junior ${ }^{10000-0002-8503-9275]}$ and Lucy Yoshida ${ }^{2}$ \\ ${ }^{1}$ Industrial and Systems Engineering Graduate Program, Pontifical Catholic University of \\ Parana, Curitiba, Brazil \\ \{matheus.canciglieri, anderson.szejka, \\ osiris.canciglieri\} epucpr.br \\ ${ }^{2}$ NHS Electronic Systems, Curitiba, Brazil \\ lucyenhs.com.br
}

\begin{abstract}
The competitive environment in which the manufacturing industries are inserted ensures that to achieve competitiveness the businesses must react quickly to change and understand the balance of possible options when making complex decisions. For a high quality, timely decision across a range of complex factors, it is necessary high-quality information and knowledge available at the time of the decision making. However, each business sector requires its own view on the enterprises information it needs. This represents a problem as current software solution provide local support but do not provide trans-disciplinary interoperability that is critical to long term competitiveness. The exploration of semantic technologies has the potential to solve this problem using formalizations to share the knowledge across multi-domain environments, these systems can provide more comprehensive solutions than the approaches employed to date. The aim of this paper is to study the current issues regarding the application of ontology-driven interoperability for Product Development and Manufacture. This objective will be achieved through a literature review on Semantic Interoperability, Semantic Rules and Multiple Domains and a Discussion on the current issues of each topic. This research showed that the knowledge enrichment introduced by knowledge translation models aids the creation of an interoperable environment between the product design and its manufacture.
\end{abstract}

Keywords: Semantic Interoperability, Semantic Rules, Multiple Domains, Product Design and Manufacture.

\section{Introduction}

In recent years the trend of product design is towards the formation of business collaborative alliances between enterprises and as consequence the manufacturing companies have been focusing on a more knowledge intensive product development 
process with the aim of reduce the overall product design time and increase its activities efficiency. To achieve this aim, the enterprises relay more heavily in emerging computer network technologies, such as, the Semantic Web [1][2][3].

The Semantic Web is a web of relations among entities, therefore its role is to operate as a virtual model base in a way where the product design collaborators can have ubiquitous access to the product model, through a client interface, while understanding the semantic and context of the information.

Interoperability, a systemization of knowledge, is one of the key aspects of the semantic web and is reached through ontological integration with the aim to create a common ontology for all the information sources in a scenario of information exchange [4]; [5];[6];[7].

An ontology is an explicit, formal specification of terms in a domain and the relations between these terms. [8], that is, an ontology is a data structure of a knowledge domains concepts and how they relate with each other, in a machine-readable format [9] [27].

The knowledge from each node within the product development and manufacture may be formalized which will result in multiple viewpoints associated to the representation of artifacts and different depictions of similar concepts [10] [27].

To [11], these multiple representations of an artifact or a concept is known as semantic heterogeneity, which is an obstacle to semantic interoperability.to solve this problem the process of semantic reconciliation is used. This process is traditionally made by hand by the database administrator or a designer due to its complexity. However, with the introduction of the semantic web this process shifts towards a more automated environment.

Therefore, the aim of this paper is to is to study the current issues regarding the application of ontology-driven interoperability for Product Development and Manufacture in order to provide sustenance to a proposal of an ontology-driven semantic reconciliation model that aims to use ontology mapping techniques to aid the product design and manufacture processes knowledge share

\section{Problem Statement}

Globalization, collaboration and cooperation have contributed to the emergence of a knowledge sharing culture in open and large environments [12] [13] [27]. However, communication between project teams is often hindered by the lack of clarity in the terms used. This represents that the context in which the information is exchanged affects the overall meaning and interpretation of the shared implicit and explicit knowledge. Therefore, two problems may appear: I) the same term is being applied to different concepts (semantic problem) and II) different terms are being applied to the same concept (syntax problem) [14]. These problems are known as semantic heterogeneity

One approach to solve these problems, is the creation of an ontology in which a specific domain's concepts and terms are defined and can be understood through the different teams across that domain. Nevertheless, this presents a different issue: How 
can the knowledge be shared effectively through multiple domain systems without the problems caused by semantic heterogeneity?

The use of different ontology throughout the product design and manufacture may hinder the creation of an interoperable environment as the different domains have different concepts and the exchange of this information through the product development without the proper processing may result in loss of product quality, greater development time and costs.

\section{Current Issues}

The current issues were researched and structured according to the main topics related to the research objective: i) Semantic Interoperability; ii) Semantic Reconciliation; iii) Semantic Rules.

\subsection{Semantic Interoperability}

Even though the product development process presents a holistic approach to provide the necessary information to the different phases of the product design and manufacturing, it has been identified misinterpretations and mistakes during the latter stages of the product development [15]. These mistakes become more significant when the activities of the design and manufacture cost $85 \%$ of the products final cost [16]. Consequently, the information sharing across the different stages of product development and manufacture must be done efficiently to ensure that the product developed has the desired quality with cost and time optimization.

This is a semantic interoperability problem for which the meaning associated to the captured information must be shared across different domains inside a system without any loss of meaning and intent during the exchange process [17]. The most common method to ensure that there is no loss of meaning in the information exchange process has been the definition of common information models [18] [19]. In this context, the construction of ontologies is a viable solution on the formalization of these common information models and on the sharing of the formal information throughout the stages of the product development process, which, consequently, provides increased knowledge in the domains of application. [19] [20]

An Ontology is defined as "a lexicon of specialized terminology along with some specification of the meaning of terms on the lexicon" [21], where the lexicon is the vocabulary of a knowledge domain. Therefore, an important differentiation can be made between ontologies by their degree of expressiveness. In this differentiation, simple ontologies, which formalizes only a taxonomy of concepts and basic relations between them are referred as lightweight ontologies. When a lightweight ontology is enriched through the insertion of axioms in the form of constraints, they are classified as a heavyweight ontology. Nevertheless, the use of ontologies is restricted to the purpose of its application, that is, the knowledge structure formalized in an ontology has little reusability outside the scope of its application. [17] 
Despite the semantic formalism created using ontologies, a limitation appears when the need to work in multiple knowledge domains is presented, as the semantic formalism of the ontology cannot ensure the sharing of the information and its meaning through different domains. However, this problem is moderated with the development of ontology mapping methodologies, which can create relationships between terms in different ontologies of different domains. [16]

\subsection{Semantic Reconciliation}

The increase in the perception that to make better decision, it is essential to have usable ou actionable information, which can be defined as knowledge, in an integrated environment between diverse resources. Therefore, the importance of resolving semantic heterogeneity has gained attention in various domains [22] [17].

The emerging semantic web, which is a specific form of formal logic that can be used efficiently in a virtual environment, has used descriptive logic based ontologies as one of its primary applications. These ontologies can take advatange of better expressive constructs, however, when used in a multiple domain environment they suffer from limitations to share the knowledge efficiently between them. [22] [16] [17]

For example, consider two different classification systems, a simple query is finding all the data conrresponding to a term in both information sources, however, this query can only be efficiently answerd if both of the systems have their semantics well understood. If these systems are conceptualized in two different ontologies the comparation of terms is a challenge due to the great variation of the detail level and logic between these ontologies. [22] [17].

To solve this limitation a shared ontology approach can be adopted. This approach enables terminological reasoning over the definition of classes in the descriptive logic ontologies by considering the axioms, set of relations and set of class definitions defined in the shared ontology [23] [26]

Even with the considerable effort that has been input to address the obstacles of semantic interoperability brought by semantic mismatches, there are still several challenges to improve the semantic reconciliation techniques[25] [26] Nowadays, the most popular techniques are the ontology mapping/matching.

Initial work on these techniques focused mainly on the overall nomenclature of the ontologies, which lead to various conflicts in a multiple domain environment or even in similar domain ontologies that uses different taxonomies. [25]. However ontology mapping techniques have been a key direction to solve semantic heterogeneity issues using the reconciliation of the semantics of the ontology based models. Even though, several perspectives of the ontology mapping methods have been proposed, there is a consensus over the types of methods that can be applied to do a ontology mapping/matching. Figure 1 presents these methods [2] [10]. 


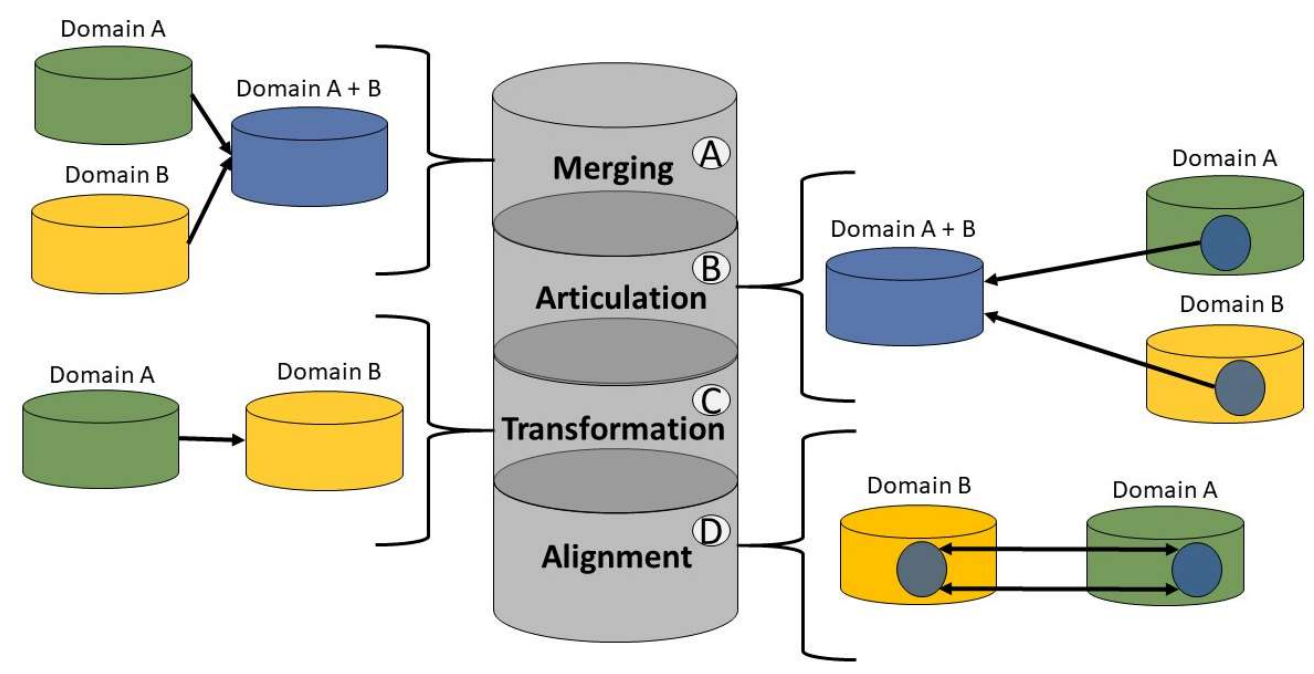

Fig. 1. Ontology mapping/matching techniques categorization. Adapted from [10].

Ontology mapping methods can be classified as one of 4 categories or as hybrids of these. The ontology mapping categories are, as shown in figure 1: i) techniques that merge two ontologies to construct a new ontology from the individual ontologies (Detail A); ii) methods that through a transformation function, transforms a given ontology into another based on the rules specified(Detail C); iii) techniques that establish binary relations between the vocabularies of two ontologies (Detail B) and IV) methods that enable specific protions of two ontologies to be reconciled with the use of semantic mappings made through an articulation ontology (Detail D) [10] [2] [17].

Even though ontology mapping/matching has been key to solve semantic heterogeneity problems, there is presently of methdos that rely on lexical similarity matching, which is not optimal from a semantic interoperability viewpoint. This is because in a multiple domain environment similar terms are used across different groups to refer to diverse concepts. Ergo, it is only through the semantics associated to these terms that existing differences can be identified, highlighting the need to capture semantics in the first place [10]

On the other hand, ontological formalisms lite the Web Ontology Language (OWL) support built-ins for ontology mapping, however, these built-ins have limitions when mapping the semantic content of manufacturing ontologies and their associated knowledge bases [10] [17] [26].

Moreover, there are requirements to aid the construction of mapping/matching techniques which can be formally interpreted and are focused at inditifiying potential solutions for semantic mismatches. Therefore, enabling the reconciliation process at several levels, including the instance level, of ontology based models. These requirements aid the reconciliation process to have better accuracy, automation and reduces the time that takes to resolve cross-model correspondences [17] [25]. 


\subsection{Semantic Rules}

The web ontology language (OWL) relies only on description logic, however both description logic and rules are required for a semantic web application because they can overcome expressiveness limitations through extensions of different knowledge domains. Nevertheless, each paradigm supports specific reasoning services and for them to work efficiently there is a need to a close integration between the description logic and semantic rules [3].

The semantic web rule language (SWRL) extends the description logic of the OWL with the ability to write rules and permiting the addition of horn logic rules to the OWL descriptions. This characteristic allows the construction of more complex relations and can be used to define more precisely the concepts in the ontology. The SWRL rules are an implication between an antecedent and a consequent that can be read as when the conditions specified in the antecedent are true then the conditions on the consequent must also be true. [3] [4] [24]

Although the SWRL is supported by the Protégé ontology editor and by several rule inference engines such as Pellet, it has been around fore more than 10 years making it very unlikely that it will become a $\mathrm{W} 3 \mathrm{C}$ standard, therefore difficult to reach out to the industrial world. [24]

An alternative to the SWRL is the SPARQL Inferance Notation (SPIN), which has become an industrial standart and uses representations of the SPARQL inqueries to construct rules and constraints in semantic web models. SPIN cobines concepts from object oriented languages, query languages and rule based systems to describe objects behaviors in the semantic web, that is, it links class definitions with queries in order to capture constraints and rules that will formilize the expected behavior of those classes.

Since SPIN is represented in RDF, the rules and constraints can be shared with the class definitions they are associated with. This attachment of rules to the classes they refer to aids in the maintenance of the database.

The SPIN vocabulary defines several properties that can be used to attach SPARQL queries to the classes and the reasoning engines will construct inferred RDF triples from the currently asserted information in the model. These queries are interpreted in the context of the classes to which they refer to. [24]

The use of SPIN to create the constraints and relationships of the concepts described in a semantic web model allows it to have an object oriented view in which the SPARQL queries play similar role to the functions and methods. The Inheritance is treated in the sense that any rule defined for super classes will also be applied to the subclasses. This means SPIN rules can only further restrict what has been defined in the uuper classes of the model. [24] 


\section{Discussion}

This research works towards finding evidence that demonstrates the current issues regarding semantic interoperability, semantic reconciliation and semantic rules in a multiple domain environment. These issues provide sustenance to the proposal of an ontology-driven semantic reconciliation model that aims to use ontology mapping techniques to aid the product design and manufacture processes knowledge share, therefore, ensuring higher quality products and cost reduction of the product design steps and its manufacture. Additionally, the model aims uses semantic rules to better simulate the behaviour of the concepts thus optimizing the reconciliation and translation process of the model.

The mapping of the current issues revealed the limitations of a ontology driven interoperability system when it is used in a multiple domain environment, and, also, how the sharing of ambiguous information in such an environment can reduce the competitiveness of an enterprise as it will raise the costs and diminish its products quality.

In order to ensure a semantic interoperability environment across multiple domains, ontology mapping/matching techniques are necessary as through them it is possible to create a multiple domain ontology that contains all the relevant information to the domains and has translation mechanisms which will translate the concepts within this ontology to all other domains. However, these techniques are made mostly by hand which makes them expensive and time demanding. This makes their use in industrial applications limited.

The semantic rules aid in the creation of a semantic interoperable environment as they create more complex class expressions and enable the simulation more accurate of the concepts behaviour and, therefore, infere the behaviour of similar concepts integrating the different knowledge domains. Yet there are limitations to their use as there is not a method to conceive these rules outside the determination of an expert, which makes their creation an expensive and long process. the main language to the development of semantic rules, SWRL, has some limitations that are being solved through SPARQL based rule languages as SPIN.

During the product development and manufacture the departaments envolved must manage their resources and at the same time need to communicate, cooperate efficiently with the other teams in the process. Therefore the issues presented in this research represent the biggest problems that interfere with the application of an efficiently multiple domain communication and cooperation system that creates a true knowledge share environment which reduces the product's development and manufacture time, cost and ensures its quality.

Thus, the essential requirements for effective and efficient collaboration among enterprises are agility and interoperability

Through the analisys of multiple domains and their semantic constrains throughout the product development process, a model can be developed which represents the points where the semantic constrains and rules may hinder the development of an interoperable environment as can be seen in the Figure 2. 
Detail A represents the possibility of ambiguous constraints present in a same domain and same product design stage. Detail B shows the possibility of having heterogenious constraints in a same domain but in different stages of the product development process. Detail $\mathrm{C}$ characterises the heterogeneity between constraints in the same development stage, however, between different knowledge domains. Finally, Detail D represents the heterougeneity between constrains in different stages of the product design and in different domains.

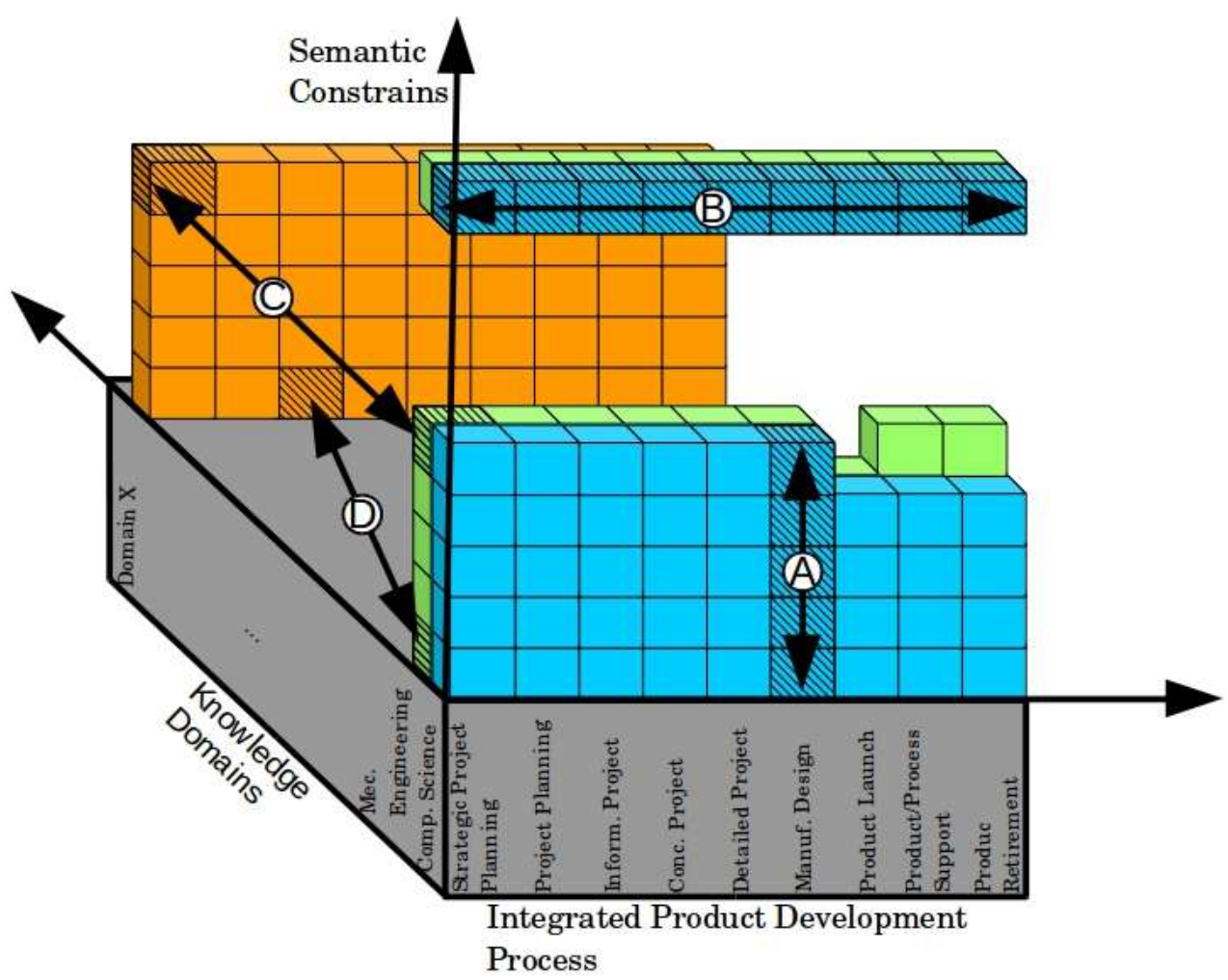

Fig. 2. Diagram of Interoperable Limitations Regarding Product Deign and Manufacture, Multiple Domains and Semantic Constrains.

\section{Conclusion}

This paper presented a research that lead to the development of a model which shows some of the critical issues that hinder the development of a true ontology-driven interoperable environment between product design and manufacture. These areas are essential to ensure a competitive position for an enterprise in the global competitive environment it is inserted. 
The standardized and formilised knowledge that is captured by an ontology driven system allows it to be retrieved, shared and reused in different stages of the product development and manufacture and, also, through the process of relating concepts made in the ontology design the information can be captured in its enterity as well as extended as the need arrises. This integration improves the collaboration in a multiple domain environment and across network based designs as it conveys several characteristics, that are often ambiguous, in a non-ambiguous manner. These knowledge structures can, also, be used to create optimization models.

The high degree of expressiveness of a ontology-driven structure enables the establishment of resolvable and meaningful mappings across knowledge models which help support the consistency of the ontology matching while also avoiding the drawbacks of subjectivity in the mapping transaction that are a consequence of extensive human intervention.

In order to continue this research several subjects are proposed for future works: Research semantic rules development methods for ontology mapping/matching; Map product development and manufacture structures and important concepts for the development of reference ontologies which can be then specialized to specific projects; Research and define a Semantic Reconciliation Method for Multi-Domain Environments.

\section{References}

1. Engineous. Engineous Int. Symp.Workshop, Novi,Mi,October 10-12 (2005).

2. Kyoung-Yun Kim,Seongah Chin, Ohbyung Kwon, R. Darin Ellis. Ontology-Based Modeling And Integration Of Morphological Characteristics Of Assembly Joints For NetworkBased Collaborative Assembly Design. Artificial Intelligence For Engineering Design, Analysis And Manufacturing, 23, 71-88 (2009).

3. W. Zhao, J.K. Liu Owl/Swrl Representation Methodology For Express-Driven Product Information Model Part I. Implementation Methodology. Computers In Industry, 59, 580-589 (2008).

4. Biletskiy, Y., O. Vorochek And A.Medovoy Building Ontologies For Interoperability Among Learning Objects And Learners, Lecture Notes In Computer Science, 3029, $977-$ 986 (2004).

5. Mizoguchi, R. Tutorial On Ontological Engineering Part 1: Introduction To Ontological Engineering. New Generation Computing 21, 4,365-384. (2003).

6. Mizoguchi, R. Tutorial On Ontological Engineering Part 2: Ontology Development Tools And Languages. New Generation Computing 22, 2, 61-96. (2004).

7. Mizoguchi, R. Tutorial On Ontological: Engineering Part 3 Advanced Course Of Ontological Engineering. New Generation Computing 22(2), 198-220. (2004).

8. Gruber, T.R. A Translation Approach To Portable Ontology Specification. Knowledge Acquisition 5, 2, 199-220. (1993).

9. Fensel, D. Ontologies: A Silver Bullet For Knowledge Management And Electronic Commerce, Second Edition, Springer, (2004).

10. Chungoora, N. And Young, R.I.M., Semantic Reconciliation Across Design And Manufacturing Knowledge Models: A Logic-Based Approach. Applied Ontology, 6, 4, Pp. $295-$ 315. (2011). 
11. Gal,A., Anaby-Tavor, A., Trombetta, A., Montesi D., A Framework For Modeling And Evaluating Automatic Semantic Reconciliation.International Journal On Very Large Data Bases, 14, 1, Pp 50-67. (2005).

12. Beau S, Taouil F-T, Hassanaly P., Elaborate Knowledge Between Necessity And Opportunity. Proceedings Of The Third International Conference Of Information Systems And Economic Intelligence (Siie),Sousse,Tunisia. P. 317-30. (2010).

13. Sinderenv. Editorial: Challenges And Solutions In Enterprise Computing. Enterp Inf Syst 2, 4, 341-6. (2008).

14. Harding, J.A. And Shahbaz, M., Manufacturing System Engineering Ontology For Semantic Interoperability Across Extended Project Teams. International Journal Of Production Research, 42, 24, Pp. 5099 - 5118. (2004)

15. Penciuc, D., A. Durupt, F. Belkadi, B. Eynard, And H. Rowson. Towards A Plm Interoperability For A Collaborative Design Support System. Procedia Cirp 25: 369-376. (2014).

16. Rozenfeld, H., F. A. Forcellini, D. C. Amaral, J. C. Toledo, S. L. Silva,D. H. Alliprandini, And R. K. Scalice. Gestão De Desenvolvimento De Produtos: Uma Referência Para A Melhoria Do Processo Product Development Management: A Reference To Improve The Process. São Paulo: Saraiva, (2006).

17. Chungoora, N. And Young, R.I.M.,A Framework To Support Semantic Interoperability In Product Design And Manufacture" Phd Thesis, Loughborough University, Loughborough , Uk. (2010).

18. Canciglieri Junior, O., And Young, R. I. M. Information Mapping Across Injection Moulding Design And Manufacture Domains. International Journal Of Production Research, Vol. 41, N. 7, Pp. 4437-4462, (2010).

19. Yang, A., Braunschweig, B., Fraga, E. S., Guessoum, Z., Marquardt, W., Nadjemi, O., Paen D., Piñol D., Roux P., Sama S., Serra M., Stalker I. A Multi-Agent System To Facilitate Component-Based Process Modeling And Design. Computers \& Chemical Engineering, Vol. 32, N. 10, Pp. 2290-2305, (2008).

20. Gomez-Perez, A., O. Corcho And M. Fernandez Lopez., Ontological Engineering: With Examples From The Areas Of Knowledge Management, E-Commerce And The Semantic Web, Madrid: Springer. (2004).

21. Iso 18629, Industrial Automation Systems And Integration - Process Specification Language. (2005).

22. S.S. Durbha, R.L.King, V.P.Shah, N.H.Younan.A Framework For Semantic Reconciliation Of Disparate Earthobservation Thematic Data. Computers \& Geosciences 35, 761-773. (2009).

23. Stuckenschmidt, H., Harmelen, F.V., Information Sharing On The Semantic Web. Springer, Berlin, Heidelberg(P.276). (2005).

24. Bassiliades, N., Swrl2spin: A Tool For Transforming Swrl Rule Bases In Owl Ontologies To Object-Oriented Spin Rules. Arxiv, pp. 1-14, (2018).

25. KUMAR S.K. and HARDING J.A., Ontology mapping using description logic and bridging axioms. Computers in Industry, 64(1), pp. 19-28, (2013)

26. Song, S., Zhang, X. \& Qin, G. Multi-domain ontology mapping based on semantics. Cluster Computing. 20(4), pp 3379-3391, (2017)

27. Nagy M. and Vargas-Vera M., Multiagent Ontology Mapping Framework for the Semantic Web. IEEE Transactions on Systems, Man, and Cybernetics - Part A: Systems and Humans, 41(4) (2011) 